\title{
Análise multifatorial do perfil de idosos ativos com história de quedas
}

\author{
Multifactorial profile analysis of active older adults with history of falls
}

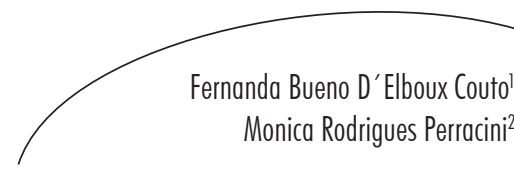

Resumo

Objetivo: Explorar o relacionamento entre variáveis sociodemográficas, clínicas, funcionais e psico-cognitivas em idosos participantes de grupo de convivência, residentes na comunidade, e identificar a associação entre a interação dessas variáveis e quedas e quedas recorrentes. Método: Estudo transversal, exploratório com 150 idosos, de ambos os sexos, participantes de um grupo de terceira idade no município de Itu, São Paulo. Utilizou-se uma entrevista semiestruturada contendo características sociodemográficas, de saúde física, físico-funcionais (Brazilian OARS Multidimensional Functional Assessment Questionnaire - BOMFAQ e Timed up and go test - TUG), de saúde mental (Geriatric Depression Scale - GDS-15 e Mini Mental State Examination - MMSE). O nível de atividade física foi determinado por questões sobre frequência e duração das atividades físicas. A variável desfecho utilizada foi o número de quedas no último ano. Foi conduzida uma análise de cluster para se identificar perfis de grupos formados pela distribuição da variabilidade dos dados em cada bloco de variáveis: sociodemográficas, desempenho físico-funcional, saúde física e mental. Procedeu-se a análise de regressão uni e multivariada com quedas únicas e quedas recorrentes. Resultados: A idade média dos participantes foi de $71,9( \pm 5,6)$ anos. A prevalência de quedas foi de 38,7\%. A análise univariada revelou uma associação independente entre o cluster saúde mental (sem sintomas depressivos e com declínio cognitivo) e quedas recorrentes ( $\mathrm{OR}=2,73$ IC 95\% 1,04-7,22 p=0,042), porém esta associação não foi significativa na análise multivariada $(\mathrm{p}=0,082)$. Conclusão: Não foi possível identificar um perfil de caidores e caidores recorrentes em idosos ativos. Mas nossos achados sugerem que, em idosos ativos com história de quedas, uma avaliação geriátrica abrangente com ênfase na função cognitiva seja considerada.

\footnotetext{
Programa de Mestrado em Gerontologia. Universidade Estadual de Campinas. Campinas, SP, Brasil.

2 Programa de Pós-graduação em Gerontologia. Universidade Estadual de Campinas. Campinas, SP, Brasil e Programa de Pós-graduação em Fisioterapia. Universidade Cidade de São Paulo. São Paulo, SP, Brasil.

Correspondência / Correspondence

Monica Rodrigues Perracini

E-mail: monica.perracini@unicid.edu.br
}

Palavras-chave: Idosos. Acidentes por quedas. Fatores de risco. Envelhecimento ativo. 


\section{Abstract}

Objective: To explore the relationship among sociodemographic, clinical, functional and psychocognitive variables in older adults who attend a community-based group, and to identify the association between the aggregate interaction of these variables and single and recurrent falls. Methods: A cross sectional exploratory study with 150 older adults, of both genders who attended a third age group, in Itu municipality, São Paulo. It was used a semi-structured interview including: sociodemographic, physical health, physical functioning (Brazilian OARS Multidimensional Functional Assessment Questionnaire - BOMFAQ, Timed up and go test-TUG) and mental health (Geriatric Depression Scale - GDS-15; Mini Mental State Examination - MMSE). Physical activity level was determined by questions about frequency and duration of physical activities. The outcome variable was the number of falls in the previous year. A cluster analysis was conducted to search for profiles of groups composed by the joint variation in variable distribution regarding sociodemographic, physical functioning, physical and mental health data. Univariate and multivariate logistic regression analyses were used to identify association between socio-demographic, physical functioning, physical and mental health clusters and single falls and recurrent falls. Results: The mean age was 71.9 (5,6 SD) years. The univariate analysis revealed an association between the mental health cluster (without depressive symptoms but with cognitive decline) and recurrent falls $(\mathrm{OR}=2.73 \mathrm{IC} 95 \%$ 1.04-7.22 $\mathrm{p}=0.042)$ but this association was not significant in the multivariate analysis $(\mathrm{p}=0.082)$. Conclusion: It was not possible to identify any profile of single fallers and recurrent fallers among active older adults. However, our findings suggest that in active older fallers, a comprehensive geriatric assessment, with a focused approach on cognitive function should be considered.

Key words: Elderly. Accidental falls. Risk factors. Active ageing.

\section{INTRODUÇÃO}

Estimativas apontam para um aumento dos custos relacionados ao tratamento das lesões por quedas de forma dramática, tendo em vista o aumento da proporção de idosos na população e o aumento da expectativa de vida. ${ }^{1}$ Cerca de $30 \%$ a $40 \%$ dos idosos com 65 anos ou mais que vivem na comunidade caem uma vez ao ano, e estas taxas aumentam de forma expressiva a partir dos 75 anos. Metade destes idosos cai ao menos duas vezes ao ano. Essas prevalências possivelmente estão subestimadas devido ao viés de memória e a tendência dos idosos em sub-relatar os eventos que não tenham causado lesões aparentes. ${ }^{2-4} \mathrm{Os}$ acidentes são a quinta causa de morte entre os idosos e as quedas são responsáveis por dois terços destas mortes acidentais. ${ }^{5}$

Estudos apontam que a prevalência de quedas é alta tanto em idosos ativos e saudáveis, ${ }^{6}$ quanto naqueles idosos com menor nível de atividade e mais frágeis. ${ }^{7}$ No primeiro grupo, a ocorrência de quedas está relacionada à maior exposição a fatores de risco ambientais e a tarefas com maior demanda sobre o equilíbrio corporal, enquanto nos frágeis o peso dos fatores de risco intrínsecos, como fraqueza muscular, distúrbios do equilíbrio corporal e da marcha, seria mais determinante. Idosos que caem em ambiente externo gastam mais tempo em atividades recreativas, têm melhor saúde e melhor funcionalidade do que idosos que caem dentro de casa. ${ }^{8-10}$ No entanto, parece ainda incerto qual o peso do nível de atividade na determinação de uma maior chance de cair em diferentes populações. ${ }^{11}$

Recente estudo, ${ }^{12}$ com seguimento por 12 meses de uma coorte de 500 idosos, identificou uma prevalência de $33,2 \%$ de quedas. A análise multivariada baseada numa árvore de classificação identificou que os idosos com bom desempenho em testes físicos em sistemas que envolvem o equilíbrio corporal, mas com algum grau de incapacidade (limitação da mobilidade, dor ou depressão) podem ser divididos em dois grupos: aqueles que tinham níveis intermediários de atividade e aqueles que não faziam nenhuma 
atividade ou faziam quatro ou mais horas de atividade física planejada. Este último grupo tem maior risco de queda $(36,8 \%$ vs $21,4 \%)$, demonstrando que a atividade física pode não ter uma relação linear com queda, expondo tanto idosos com baixos níveis quanto aqueles com altos níveis de atividade. ${ }^{12}$

É sabido que há um aumento significativo do risco de cair com o aumento do número de fatores de risco individuais presentes. ${ }^{13}$ Em um estudo, o risco anual de queda aumentou de $8 \%$ em idosos sem nenhum fator de risco presente para $78 \% \mathrm{em}$ idosos com quatro ou mais fatores presentes. ${ }^{7,13}$ Vários fatores de risco têm sido destacados em estudos com idosos que vivem na comunidade, entre eles: quedas prévias, disfunções de equilíbrio, fraqueza muscular, limitações visuais, uso de quatro ou mais medicações e uso de medicação psicotrópica, dificuldade para andar, depressão, tontura ou hipotensão ortostática, incapacidade em atividades básicas de vida diária, idade avançada, gênero feminino, comprometimento cognitivo e incontinência urinária, dentre outros. ${ }^{7,14,15}$ Destes, os apontados como de maior evidência na determinação do risco são: comprometimento cognitivo, uso de medicação psicotrópica e polifarmácia, presença de riscos ambientais, problemas de visão, fraqueza muscular ou disfunção em MMII, diminuição da função neuromuscular periférica, incapacidade em atividades básicas e instrumentais de vida diária e uso de dispositivos de auxílio à marcha. ${ }^{15}$

No entanto, mais do que o peso da presença de cada fator de risco, a interação entre eles parece ser de grande relevância. ${ }^{16} \mathrm{O}$ entendimento das inter-relações e associação entre variáveis apontadas na literatura como indicadores de "risco" de quedas possibilita a identificação de agrupamentos que caracterizem melhor os perfis presentes em populações específicas, como as de grupos de idosos que frequentam grupos de convivência. A identificação de perfis pode colaborar no desenvolvimento de avaliações e intervenções multidimensionais.

O objetivo deste artigo é explorar o relacionamento de variáveis sociodemográficas, clínicas, funcionais e psico-cognitivas em idosos participantes de grupo de convivência, residentes na comunidade, e identificar a associação entre a interação dessas variáveis e quedas e quedas recorrentes.

\section{MÉTODOS}

Trata-se de estudo transversal exploratório, com uma amostra de conveniência, de idosos ativos participantes de um grupo de convivência, aprovado pelo Comitê de Ética da UNICAMP, sob o protocolo número 531/2005.

Foram critérios de inclusão: ter 65 anos ou mais; ser residente no município de Itu-SP; ser inscrito no Grupo da Melhor Idade e participar sistematicamente das atividades nos 12 meses anteriores a pesquisa. Foram excluídos os idosos com comprometimento cognitivo, que não eram capazes de andar mesmo que com uso de dispositivo de auxiliar da marcha, e aqueles que residiam em instituição de longa permanência.

O comprometimento cognitivo foi avaliado por meio do Mini-Exame de Estado Mental ${ }^{17,18}$ conforme a seguinte pontuação: ${ }^{17} 17$ para analfabetos, 22 pontos para idosos com escolaridade entre 1 e 4 anos, 23 para idosos com escolaridade entre 5 e 8 anos e 26 para aqueles com 9 anos ou mais de escolaridade.

Dos 1.227 participantes inscritos, 683 idosos foram pré-selecionados. Após a verificação dos critérios de exclusão, 150 idosos foram, por conveniência, incluídos na amostra, sendo 128 mulheres e 22 homens.

A variável desfecho considerada neste estudo foi ter caído nos últimos 12 meses, e para caracterização da amostra criaram-se três categorias: "idosos que não caíram", "idosos que caíram uma vez" e "idosos que caíram duas ou mais vezes", denominados de "idosos com queda recorrente". Queda foi definida como "evento inesperado no qual a pessoa venha a repousar no chão, ou em um nível inferior”." ${ }^{9}$

As variáveis selecionadas para compor o estudo foram divididas nas seguintes dimensões: 
sociodemográficas (idade, sexo, estado civil, escolaridade), saúde física (doenças crônicas referidas que foram diagnosticadas por médico nos últimos 12 meses, número de medicamentos, uso de medicamentos psicoativos, história de internação no último ano, medo de cair, queixa de tontura e fraqueza, ou alteração de sensibilidade em membros inferiores, peso e estatura corporal e percepção subjetiva de saúde, de visão e audição), físico-funcionais (atividades de vida diária - AVD, atividades instrumentais da vida diária - AIVD, mobilidade, atividade física) e saúde mental (sintomas depressivos e declínio cognitivo). Foram coletados dados sobre história e consequência de quedas no último ano.

Foram utilizados os instrumentos Timed Up and Go Test (TUGT) para avaliação da mobilidade, ${ }^{20}$ a Escala de Depressão Geriátrica abreviada (Geriatric Depression Scale -GDS $)^{21}$ em sua versão curta, de 15 itens, validada para a população brasileira, ${ }^{22}$ - BOMFAQ (Brazilian OARS Multidimensional Functional Assessment Questionnaire), que avalia a capacidade de realizar 15 atividades de vida diária (AVD) e atividades instrumentais de vida diária (AIVD). ${ }^{23}$ A visão e audição foram avaliadas por meio da percepção subjetiva. As variáveis "peso corporal" e "estatura" foram coletadas e adotou-se a graduação proposta por Lipschitz, ${ }^{24}$ no qual o IMC entre $22-27 \mathrm{~kg} / \mathrm{m}^{2}$ indica eutrofia; maior que $27 \mathrm{~kg} / \mathrm{m}^{2}$ indica obesidade e menor que $22 \mathrm{~kg} / \mathrm{m}^{2}$ indica desnutrição. A queixa de fraqueza muscular ou alteração de sensibilidade de membros inferiores foi feita por autorrelato.

Realizou-se a análise descritiva para caracterização geral da amostra estudada. A seguir, procedeu-se a análise de cluster e análise de regressão logística univariada, seguida de um modelo multivariado saturado - com todos os clusters - para verificar a relação conjunta dos conglomerados com a variável resposta (queda única e quedas recorrentes: duas ou mais quedas). Para a análise de cluster, optou-se pela utilização do método de partição, estabelecendo a priori a criação de três grupos ou conglomerados para cada bloco de variáveis, devido ao grande número de variáveis da amostra. Nas análises, só foram utilizadas as variáveis com maiores frequências em cada categoria de resposta; com isso as variáveis "presença de doença da tiróide", "presença de DPOC" e "uso de dispositivo de auxílio à marcha" foram excluídas da análise. A variável "pratica atividade física" foi incluída na variável "frequência semanal de atividade física" para fins da análise.

Os clusters foram denominados da seguinte forma: cluster sociodemográfico (SD), cluster clínico (C), cluster funcional (F) e cluster psicocognitivo (PC). $\mathrm{Na}$ análise de regressão univariada e multivariada, foram escolhidos os clusters de referência, estabelecidos como aqueles que continham atributos de menor risco de quedas, a saber:

- Cluster sociodemográfico (SD2): sexo masculino; idade de 70-74 anos; estado civil sem vida conjugal.

- Cluster clinico (C2): sem doenças ortopédicas ou reumatológicas e sem artrose de membros inferiores; sem doenças cardiovasculares e sem hipertensão arterial; não usando nenhum ou apenas um medicamento; sem uso de drogas psicoativas; no peso ideal; sem internação nos últimos 12 meses e sem doença que limita a articulação.

- Cluster funcional (F2): praticante de atividade física com frequência de cinco ou mais vezes na semana; não sente fraqueza nas pernas; com bom desempenho no teste de equilíbrio (10 segundos ou mais) e no teste de mobilidade (em até 10 segundos); e sem dificuldade nas atividades de vida diária.

- Cluster psico-cognitivo (PC2): sem depressão e sem alteração cognitiva.

Os clusters ficaram assim constituídos: Cluster SD1: sexo feminino; 75-79 anos ou $\geq 80$ anos de idade; sem vida conjugal; Cluster SD2: sexo masculino; 70-74 anos de idade; com vida conjugal; Cluster SD3: sexo feminino; 65-69 anos de idade, sem vida conjugal; Cluster PC1: sem depressão e com alteração cognitiva; Cluster PC2: sem depressão e sem alteração cognitiva; Cluster PC3: com depressão e sem alteração 
cognitiva; Cluster C1: com doenças ortopédicas ou reumatológicas, mas sem artrose de membros inferiores; com doenças cardiovasculares e com hipertensão arterial; usando dois, três, quatro ou mais medicamentos e também fazendo uso de drogas psicoativas; no peso ideal; com internação nos últimos 12 meses e sem doença que limita a articulação; Cluster C2: sem doenças ortopédicas ou reumatológicas e sem artrose de membros inferiores; sem doenças cardiovasculares e sem hipertensão arterial; não usando nenhum ou apenas um medicamento; sem uso de drogas psicoativas; no peso ideal; sem internação nos últimos 12 meses e sem doença que limita a articulação; Cluster C3: com doenças ortopédicas ou reumatológicas e com artrose de membros inferiores; com doenças cardiovasculares e com hipertensão arterial; usando dois, três, quatro ou mais medicamentos mas sem uso de drogas psicoativas; obeso; com internação nos últimos 12 meses e com doença que limita a articulação; Cluster F1: não praticante de atividade física ou que pratica de uma a quatro vezes na semana; não sente fraqueza nas pernas; com execução do teste de equilíbrio e marcha em até dez segundos; e sem dificuldade nas atividades de vida diária; Cluster F2: praticante de atividade física com frequência de cinco ou mais vezes na semana; não sente fraqueza nas pernas; com execução do teste de equilíbrio e marcha em até dez segundos; e sem dificuldade nas atividades de vida diária; Cluster F3: não praticante de atividade física; sente fraqueza nas pernas; com execução do teste de equilíbrio e marcha em mais de dez segundos; e com dificuldades nas atividades de vida diária.

A análise foi realizada por meio do programa SPSS for Windows versão 13.0, e o nível de significância adotado foi de $\mathrm{p}<0,05$.

\section{RESULTADOS}

A amostra total foi constituída por 150 sujeitos, com idade média de $71,51( \pm 5,6)$ anos e idade máxima de 90 anos. A amostra foi composta na sua maioria por mulheres $(85,3 \%)$ e por sujeitos entre 65 e 74 anos (66,7\%).

As quedas estavam presentes em $38,7 \%$ da amostra e desses $14,7 \%$ eram caidores recorrentes. O medo de cair mostrou-se presente em 64\% dos entrevistados e, destes, 58,3\% não haviam caído. Dentre as consequências pós-queda mais relatadas, estavam a dor com necessidade de repouso $(10,3 \%)$ e a presença de hematoma, corte e/ou edema (17,2\%). Não houve ferimentos em $41,4 \%$ dos indivíduos que caíram e $67,2 \%$ não procuraram serviço médico após a queda. A tabela 1 descreve as características sociodemográficas, clínicas e psico-cognitivas da amostra.

Tabela 1 - Caracterização da amostra de idosos em grupo de convivência quanto a quedas e quedas recorrentes, no município de Itu, São Paulo, 2012.

\begin{tabular}{|c|c|c|c|c|c|c|c|c|}
\hline & \multicolumn{2}{|c|}{$\begin{array}{l}\text { Idosos que } \\
\text { não caíram }\end{array}$} & \multicolumn{2}{|c|}{$\begin{array}{l}\text { Idosos que } \\
\text { caíram } 1 \text { vez }\end{array}$} & \multicolumn{2}{|c|}{$\begin{array}{c}\text { Idosos que caíram } \\
2 \text { ou + vezes }\end{array}$} & \multicolumn{2}{|c|}{ Total } \\
\hline & $\mathrm{n}$ & $\%$ & $\mathrm{n}$ & $\%$ & $\mathrm{n}$ & $\%$ & $\mathrm{n}$ & $\%$ \\
\hline \multicolumn{9}{|l|}{ Sexo } \\
\hline Masculino & 17 & 77,3 & 03 & 13,6 & 02 & 9,1 & 22 & 14,7 \\
\hline Feminino & 75 & 58,6 & 33 & 25,8 & 20 & 15,6 & 128 & 85,3 \\
\hline \multicolumn{9}{|l|}{ Faixa etária } \\
\hline 65-69 anos & 40 & 69,0 & 10 & 17,2 & 08 & 13,8 & 58 & 38,7 \\
\hline 70-74 anos & 25 & 59,5 & 13 & 31,0 & 04 & 9,5 & 42 & 28,0 \\
\hline 75-79 anos & 17 & 50,0 & 10 & 29,4 & 07 & 20,6 & 34 & 22,7 \\
\hline 80 anos e mais & 10 & 62,5 & 03 & 18,8 & 03 & 18,8 & 16 & 10,7 \\
\hline
\end{tabular}




\begin{tabular}{|c|c|c|c|c|c|c|c|c|}
\hline & \multicolumn{2}{|c|}{$\begin{array}{l}\text { Idosos que } \\
\text { não caíram }\end{array}$} & \multicolumn{2}{|c|}{$\begin{array}{l}\text { Idosos que } \\
\text { caíram } 1 \text { vez }\end{array}$} & \multicolumn{2}{|c|}{$\begin{array}{l}\text { Idosos que caíram } \\
2 \text { ou + vezes }\end{array}$} & \multicolumn{2}{|c|}{ Total } \\
\hline & $\mathrm{n}$ & $\%$ & $\mathrm{n}$ & $\%$ & $\mathrm{n}$ & $\%$ & $\mathrm{n}$ & $\%$ \\
\hline \multicolumn{9}{|l|}{ Autopercepção da visão } \\
\hline Péssima/ruim & 33 & 63,5 & 14 & 26,9 & 05 & 9,6 & 52 & 34,7 \\
\hline Boa/excelente & 59 & 60,2 & 22 & 22,4 & 17 & 17,3 & 98 & 65,3 \\
\hline \multirow{2}{*}{\multicolumn{9}{|c|}{$\begin{array}{l}\text { Número de medicamentos em } \\
\text { uso }\end{array}$}} \\
\hline & & & & & & & & \\
\hline Nenhum & 20 & 58,8 & 07 & 20,6 & 07 & 20,6 & 34 & 22,7 \\
\hline 1 medicamento & 16 & 59,3 & 08 & 29,6 & 03 & 11,1 & 27 & 18,0 \\
\hline 2 ou 3 medicamentos & 41 & 66,1 & 15 & 24,2 & 06 & 9,7 & 62 & 41,3 \\
\hline $4 \mathrm{ou}+$ medicamentos & 15 & 55,6 & 06 & 22,2 & 06 & 22,2 & 27 & 18,0 \\
\hline Internação nos últimos 12 meses & 11 & 68,8 & 03 & 18,8 & 02 & 12,5 & 16 & 10,7 \\
\hline Fratura anterior & 26 & 55,3 & 10 & 21,3 & 11 & 23,4 & 47 & 31,3 \\
\hline \multicolumn{9}{|l|}{ Índice de massa corpórea } \\
\hline Obeso & 46 & 65,7 & 14 & 20,0 & 10 & 14,3 & 70 & 46,7 \\
\hline Eutrófico & 37 & 56,1 & 18 & 27,3 & 11 & 16,7 & 66 & 44,0 \\
\hline Desnutrido & 09 & 64,3 & 04 & 28,6 & 01 & 7,1 & 14 & 9,3 \\
\hline Presença de HAS & 54 & 66,7 & 15 & 18,5 & 12 & 14,8 & 81 & 54,0 \\
\hline Presença de diabetes & 15 & 57,7 & 09 & 34,6 & 02 & 7,7 & 26 & 17,3 \\
\hline $\begin{array}{l}\text { Presença de doença que limita a } \\
\text { articulação }\end{array}$ & 37 & 58,7 & 16 & 25,4 & 10 & 15,9 & 63 & 42,0 \\
\hline Pratica de atividade física & 41 & 62,1 & 14 & 21,2 & 11 & 16,7 & 66 & 44,0 \\
\hline \multicolumn{9}{|l|}{ Frequência semanal } \\
\hline 1 a 4 vezes na semana & 20 & 57,1 & 07 & 20,0 & 08 & 22,9 & 35 & 53,0 \\
\hline 5 ou + vezes na semana & 21 & 67,7 & 07 & 22,6 & 03 & 9,7 & 31 & 47,0 \\
\hline \multicolumn{9}{|l|}{ TUG } \\
\hline Em até 10 segundos & 79 & 59,0 & 35 & 26,1 & 20 & 14,9 & 134 & 89,3 \\
\hline Maior que 10 segundos & 13 & 81,3 & 01 & 6,3 & 02 & 12,5 & 16 & 10,7 \\
\hline \multicolumn{9}{|l|}{ AVD } \\
\hline Sem dificuldade & 41 & 59,4 & 20 & 29,0 & 08 & 11,6 & 69 & 46,0 \\
\hline \multicolumn{9}{|l|}{ Com alguma dificuldade } \\
\hline & 51 & 63,0 & 16 & 19,8 & 14 & 17,3 & 81 & 54,0 \\
\hline \multicolumn{9}{|l|}{ Presença de depressão } \\
\hline & 16 & 55,2 & 06 & 20,7 & 07 & 24,1 & 29 & 19,3 \\
\hline \multicolumn{8}{|l|}{ Presença de alteração } & 26,7 \\
\hline Total & 92 & 61,3 & 36 & 24,0 & 22 & 14,7 & 150 & 100 \\
\hline
\end{tabular}


A tabela 2 descreve os resultados da análise de conglomerados para cada bloco de variáveis: sociodemográficas, psico-cognitivas, clínicas e funcionais. A variável "faixa etária" explicou $78,6 \%$ da variabilidade dos dados e o conjunto das três variáveis (sexo, faixa etária e estado civil) explicou $63,5 \%$. Observa-se que a variável "presença de alteração cognitiva" explica 100\% da formação dos clusters e quando se verifica o conjunto das variáveis psico-cognitivas, este cluster responde por $84,8 \%$ da variabilidade dos dados. Os resultados da análise de conglomerados para as variáveis clínicas mostram que os clusters formados são predominantemente explicados pelas variáveis "número de medicamentos em uso" $(73,1 \%)$ e "índice de massa corpórea" $(70,8 \%)$, já o conjunto de variáveis clínicas explica $28,5 \%$ da formação dos conglomerados.
Já para os blocos das variáveis funcionais, a análise de conglomerados mostrou que a variável "frequência da atividade física" explicou 74,8\% da formação do cluster, e que o conjunto das variáveis explicou $54,1 \%$ dos dados.

A associação entre os clusters contendo as variáveis sociodemográficas, psico-cognitivas, clínicas, funcionais e as variáveis dependentes "quedas" e "quedas recorrentes" foi investigada inicialmente por meio da análise de regressão logística univariada, que está apresentada na tabela 3.

A influência concorrente dos clusters com as variáveis dependentes quedas e quedas recorrentes foi explorada por meio da análise de regressão multivariada que está apresentada na tabela 4. 
Tabela 2 - Análise de conglomerados para cada bloco de variáveis: sociodemográficas, psico-cognitivas, clínicas e funcionais em idosos de grupo de convivência, município de Itu, São Paulo, 2012.

\begin{tabular}{|c|c|c|}
\hline & $r^{2}$ & $\mathrm{r}^{2}$ Total \\
\hline $\begin{array}{l}\text { Variáveis sociodemográficas } \\
\text { Sexo }\end{array}$ & 0,057 & \\
\hline Faixa etária & 0,786 & \\
\hline Estado civil & 0,257 & \\
\hline & & 0,635 \\
\hline $\begin{array}{l}\text { Variáveis psico-cognitivas } \\
\text { Presença de depressão }\end{array}$ & 0,659 & \\
\hline Presença de alteração cognitiva & 1,000 & \\
\hline & & 0,848 \\
\hline $\begin{array}{l}\text { Variáveis clínicas } \\
\text { Hipotensão ortostática }\end{array}$ & 0,005 & \\
\hline Medo de cair & 0,021 & \\
\hline Autopercepção da visão & 0,000 & \\
\hline Autopercepção da audição & 0,025 & \\
\hline Queixa de tontura & 0,006 & \\
\hline Presença de vestibulopatia & 0,007 & \\
\hline Presença de doença neurológica & 0,002 & \\
\hline Presença de sequela deAVC & 0,001 & \\
\hline Presença de doença ortopédica & 0,079 & \\
\hline Presença de artrose em membros inferiores & 0,109 & \\
\hline Presença de doenças cardiovasculares & 0,163 & \\
\hline Presença de HAS & 0,218 & \\
\hline Presença de diabetes & 0,010 & \\
\hline Presença de incontinência urinária & 0,032 & \\
\hline Número de medicamentos em uso & 0,731 & \\
\hline Uso de drogas psicoativas & 0,147 & \\
\hline Alteração da sensibilidade em membros inferiores & 0,014 & \\
\hline Índice de massa corpórea & 0,708 & \\
\hline Internação nos últimos 12 meses & 0,064 & \\
\hline Fratura anterior & 0,008 & \\
\hline \multirow[t]{2}{*}{ Presença de doença que limita a articulação } & 0,088 & \\
\hline & & 0,285 \\
\hline $\begin{array}{l}\text { Variáveis funcionais } \\
\text { Frequência semanal da atividade física }\end{array}$ & 0,748 & \\
\hline Fraqueza muscular & 0,356 & \\
\hline Equilíbrio e marcha & 0,186 & \\
\hline \multirow[t]{2}{*}{ Atividade de vida diária } & 0,312 & \\
\hline & & 0,541 \\
\hline
\end{tabular}




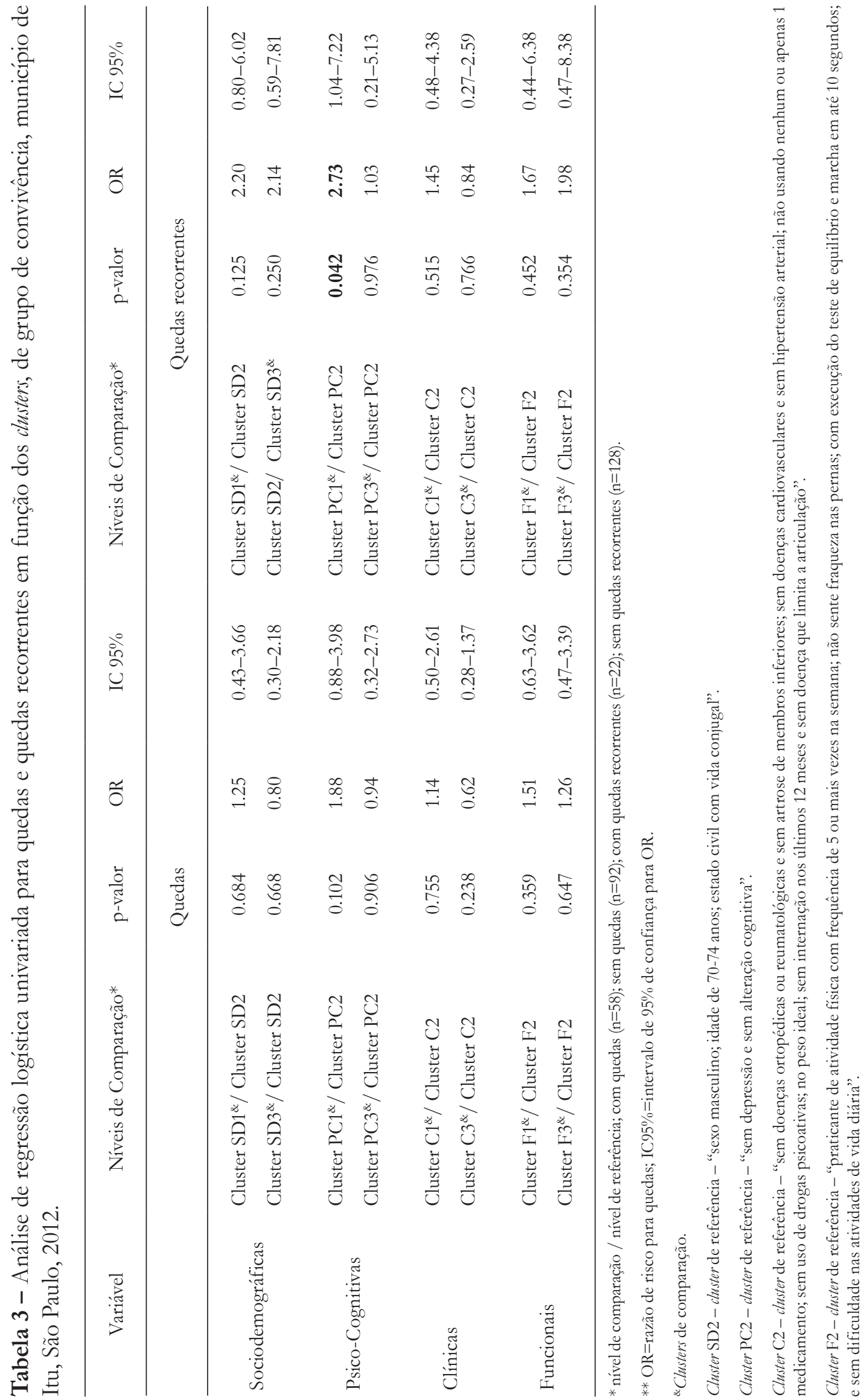


Tabela 4 - Análise de regressão logística multivariada para quedas e quedas recorrentes em função dos clusters, de grupo de convivência, município de Itu, São Paulo, 2012.

\begin{tabular}{|c|c|c|c|c|c|c|c|c|}
\hline Variável & $\begin{array}{c}\text { Níveis de } \\
\text { Comparação* }\end{array}$ & p-valor & OR & IC 95\% & $\begin{array}{c}\text { Níveis de } \\
\text { Comparação* }\end{array}$ & p-valor & OR & IC 95\% \\
\hline & & Queda & & & & edas rec & prrentes & \\
\hline \multirow{4}{*}{ Sociodemográficas } & Cluster SD1 / & & & & Cluster SD1 / & & & \\
\hline & Cluster SD3 & 0.285 & 1.52 & $0.70-3.30$ & Cluster SD3 & 0.173 & 2.06 & $0.73-5.85$ \\
\hline & Cluster SD2 / & 0.727 & 1.20 & $0.43-3.40$ & Cluster SD2 / & 0.322 & 1.97 & $0.51-7.55$ \\
\hline & Cluster SD3 & & & & Cluster SD3 & & & \\
\hline \multirow{4}{*}{ Psico-Cognitivas } & Cluster PC1 / & & & & Cluster PC1 / & & & \\
\hline & Cluster PC2 & 0.127 & 1.88 & $0.84-4.23$ & Cluster PC2 & 0.082 & 2.53 & $0.89-7.17$ \\
\hline & Cluster PC3 / & 0.886 & 1.09 & $0.36-3.30$ & Cluster PC3 / & 0.961 & 1.04 & $0.20-5.55$ \\
\hline & Cluster PC2 & & & & Cluster PC2 & & & \\
\hline \multirow{4}{*}{ Clínicas } & Cluster C1 / & & & & Cluster C1 / & & & \\
\hline & Cluster C2 & 0.779 & 1.13 & $0.48-2.64$ & Cluster C2 & 0.513 & 1.47 & $0.47-4.64$ \\
\hline & Cluster C3 / & 0.320 & 0.66 & $0.29-1.49$ & Cluster C3 / & 0.889 & 0.92 & $0.29-2.97$ \\
\hline & Cluster C2 & & & & Cluster C2 & & & \\
\hline \multirow{4}{*}{ Funcionais } & Cluster F1 / & & & & Cluster F1 / & & & \\
\hline & Cluster F2 & 0.595 & 1.28 & $0.52-3.18$ & Cluster F2 & 0.737 & 1.27 & $0.32-5.12$ \\
\hline & Cluster F3 / & 0.846 & 0.90 & $0.31-2.65$ & Cluster F3 / & 0.880 & 1.13 & $0.24-5.41$ \\
\hline & Cluster F2 & & & & Cluster F2 & & & \\
\hline
\end{tabular}

* nível de comparação / nível de referência; com quedas ( $\mathrm{n}=58$ ); sem quedas ( $\mathrm{n}=92)$; com quedas recorrentes ( $\mathrm{n}=22$ ); sem quedas recorrentes ( $\mathrm{n}=128)$. Modelo saturado, sem uso de critério de seleção.

** OR=razão de risco para quedas; IC $95 \%=$ intervalo de $95 \%$ de confiança para OR.

\section{DISCUSSÃO}

A proporção de idosos que relataram ao menos uma queda no último ano, encontrada neste estudo, foi maior do que a apresentada em outros estudos, tais como o de Siqueira et al., no qual se observou proporção de $35 \%$ de quedas em idosos atendidos na atenção básica; no de Lebrão \& Laurenti, ${ }^{25}$ que observaram ocorrência de quedas em 28,6\% dos participantes; e uma proporção de participantes relatando ter caído no último ano maior do que a identificada por Perracini \& Ramos, ${ }^{3}$ que registraram ocorrência de uma ou mais quedas em 30,9\% e 29,1\% dos idosos, no primeiro e no segundo inquérito domiciliar, respectivamente.
Uma possível justificativa para a maior prevalência de quedas é que idosos com melhor mobilidade e níveis de atividade física maiores, semelhantes aos idosos que compõem a amostra deste estudo, podem ter o risco para quedas aumentado, pois as atividades rotineiras os deixariam mais expostos aos riscos ambientais e estes tendem a se expor mais a atividades com alta demanda sobre o controle postural, como aquelas realizadas fora de casa, em ambiente externo. ${ }^{8,9,12,26-28}$

Isto também fica evidenciado quando se comparam os clusters C3 e o C2. Os idosos que apresentaram perfil com maior número de fatores de risco para quedas: idosos com doenças 
ortopédicas, reumatológicas ou cardiovasculares, incluindo hipertensão arterial, que usavam dois, três, quatro ou mais medicações, com sobrepeso ou obesidade e com história de internação no último ano apresentaram menor chance de cair do que o perfil C2, mais saudável, embora esta associação não tenha sido estatisticamente significativa. Outra possibilidade é que os idosos que se voluntariaram a participar do estudo o fizeram justamente por terem caído nos últimos 12 meses.

Ainda que sem diferença estatisticamente significante, mais de $60 \%$ dos sujeitos que apresentavam o perfil do cluster $\mathrm{F} 2$, caracterizados por terem melhor funcionalidade, não caíram. A prática regular de exercício físico pode ser considerada um fator protetor para quedas, uma vez que promove maior força e resistência muscular, melhora o controle motor e o equilíbrio corporal. ${ }^{29}$ Esses atributos dão ao indivíduo recursos fisiológicos para executar as atividades do cotidiano com maior competência. Talvez a interação entre recursos físico-funcionais, maior exposição a riscos ambientais e fatores comportamentais devam ser equacionados na determinação da chance de uma queda. Esta relação ainda é pouco estudada, uma vez que envolve a análise conjugada desses aspectos.

Esperava-se que, tanto para quedas quanto para quedas recorrentes, os clusters SD1, PC1, C3 e F3, quando comparados com os outros clusters formados a partir das mesmas variáveis, tivessem maior chance de cair, visto que nesses aglomerados está incluído o maior número de fatores de risco, segundo os dados da literatura.

Os resultados da regressão logística univariada e multivariada para quedas não mostraram associação significativa para nenhum dos clusters, exceto entre os clusters psicocognitivos e quedas recorrentes, sendo que na análise univariada o cluster PC1 (sem depressão e com alteração cognitiva) apresentou 2,7 vezes mais chance de cair do que PC2 (sem depressão e sem alteração cognitiva), mas tal associação não foi estatisticamente significante na análise multivariada.
Em um dos poucos estudos brasileiros para estimar a associação entre demência e ocorrência de quedas e fraturas entre idosos, 37,9\% dos idosos caíram no ano anterior à pesquisa e os resultados confirmam que a presença de demência contribuiu para o aumento no risco de queda seguida de fratura grave entre idosos $(\mathrm{OR}=2,0$; 1,23-3,23; $\mathrm{p}=0,04)$, independentemente de sexo, idade, estado conjugal, estado de saúde, consumo de álcool, consumo de antiácido nas últimas 24 horas e ocorrência de quedas no ano anterior à internação. ${ }^{30}$

Delbaere et al..$^{12}$ apontam que, entre idosos com alto risco de queda mensurado pelo comprometimento em sistemas fisiológicos responsáveis pelo equilíbrio corporal, a função cognitiva foi um dos fatores discriminantes para quedas múltiplas ou quedas com lesão em um ano de seguimento. A ocorrência de quedas foi maior no grupo que apresentou comprometimento na função executiva ( $42 \%$ ), quando comparada ao grupo sem alteração na função executiva (28\%).

As habilidades cognitivas incluem capacidades para regular a atenção, planejar e antecipar possíveis consequências da ação, resolver problemas, monitorar o próprio comportamento, iniciar atividades intencionalmente e inibir atividades irrelevantes e inapropriadas. ${ }^{31}$ Tem sido demonstrado que o desempenho na marcha, por exemplo, a velocidade e variabilidade dos passos estão correlacionadas com a função executiva. ${ }^{31,32}$ Idosos com desempenho ruim em testes cognitivos, embora dentro da normalidade, apresentaram velocidade de marcha diminuída ao ultrapassar obstáculos. ${ }^{33}$ Frequentemente, os indivíduos se engajam em outras atividades enquanto caminham, o que envolve atenção dividida e, com o envelhecimento, ou na presença de declínio cognitivo, a capacidade de alocar adequadamente os recursos atencionais necessários para determinada tarefa pode comprometer a habilidade dos idosos em realizar duas ou mais tarefas simultaneamente, aumentando o risco de cair. ${ }^{34,35}$

Parece haver correlação entre aumento da variabilidade dos passos na marcha e aumento de 
anormalidades vasculares subclínicas em idosos saudáveis. Esses processos degenerativos podem comprometer a regulação da marcha e passarem despercebidos, até que tais manifestações subclínicas se tornem aparentes. ${ }^{36}$

Nenhum perfil encontrado neste estudo foi capaz de discriminar adequadamente idosos com e sem história de quedas. Em uma população ativa, a variabilidade dos sujeitos é maior e o fato de a queda única não ser a expressão de problemas intrínsecos, e sim comportamentais e ambientais, pode ser uma das razões. O estudo apresenta limitações, e generalizações devem ser realizadas com cautela. O tamanho da amostra é limitado, assim como sua composição em termos da distribuição em relação ao gênero, com maior número de mulheres, que em geral predominam nos grupos de convivência para idosos..$^{37,38}$

Embora tenha sido feito um esforço no sentido de incluir todos os idosos matriculados no grupo de convivência do município onde foi realizado o estudo, isto não foi possível, tendo em vista o fato de que muitos idosos, ainda que formalmente matriculados, não frequentavam o grupo com regularidade para as atividades oferecidas e se mantinham matriculados para poder usufruir de consultas médicas periódicas oferecidas pelo programa.

O presente estudo não mensurou o nível de atividade física dos participantes a partir de um questionário adaptado e validado, tendo apenas questionado os idosos quanto a atividades físicas planejadas, o que pode ter dificultado a identificação de uma característica importante nessa população de idosos que frequentam centros de convivência, usualmente mais engajados em atividades fora de casa.

Sugere-se que estudos futuros superem essas limitações, uma vez que se espera que o número de idosos frequentando centros de convivência tenda a crescer, na medida em que aumentam a população idosa e a expectativa de vida na velhice.

\section{CONCLUSÃO}

Sugere-se que a ocorrência de quedas em idosos que frequentam grupos de convivência é alta e, portanto, ações preventivas para idosos ativos, à semelhança daquelas propostas para idosos frágeis, são igualmente importantes. Essas intervenções deveriam contemplar ações educativas, no sentido não só de informar os idosos sobre os fatores de risco para quedas, como também trabalhar no sentido de mudar atitudes e comportamentos arriscados em relação a atividades cotidianas e ao uso do ambiente.

Seria oportuno diferenciar aquele idoso ativo que cai uma única vez de forma acidental daquele que já apresenta possivelmente condições subclínicas que, ao longo do processo de envelhecimento, poderiam vir a se manifestar de forma mais contundente e os fariam converter para o grupo de idosos com maior chance de queda e de declínio da capacidade funcional. Assim, parece que uma das características hoje marcantes para o comprometimento da mobilidade que envolve equilíbrio e marcha estaria relacionada à função cognitiva, especialmente a função executiva, que deve ser mais bem explorada no futuro em estudos envolvendo quedas em idosos ativos. 


\section{REFERÊNCIAS}

1. Roudsari BS, Ebel BE, Corso PS, Molinari NA, Koepsell TD.The acute medical care costs of fallrelated injuries among the U.S. older adults.Injury 2005;36(11):1316-22.

2. Rubenstein LZ.Falls in older people: epidemiology, risk factors and strategies for prevention. Age Ageing 2006;35 Suppl 2(ii37-ii41).

3. Perracini MR, Ramos LR.Fall-related factors in a cohort of elderly community residents. Rev Saude Publica 2002;36(6):709-16.

4. Siqueira FV, Facchini LA, Piccini RX, Tomasi E, Thume E, Silveira DS, et al.Prevalence of falls and associated factors in the elderly. Rev Saude Publica 2007;41(5):749-56.

5. Rubenstein LZ, Powers CM, MacLean CH.Quality indicators for the management and prevention of falls and mobility problems in vulnerable elders. Ann Intern Med 2001;135(8 Pt 2):686-93.

6. Chan BK, Marshall LM, Winters KM, Faulkner KA, Schwartz AV, Orwoll ES.Incident fall risk and physical activity and physical performance among older men: the Osteoporotic Fractures in Men Study. Am J Epidemiol 2007;165(6):696-703.

7. Tinetti ME, Kumar C.The patient who falls: "It's always a trade-off".Jama 2010;303(3):258-66.

8. Li W, Keegan TH, Sternfeld B, Sidney S, Quesenberry CP, Jr., Kelsey JL.Outdoor falls among middle-aged and older adults: a neglected public health problem. Am J Public Health 2006;96(7):1192-200.

9. Peeters GM, Verweij LM, van Schoor NM, Pijnappels M, Pluijm SM, Visser M, et al.Which types of activities are associated with risk of recurrent falling in older persons?J Gerontol A Biol Sci Med Sci 2010;65(7):743-50.

10. Ribom EL, Grundberg E, Mallmin H, Ohlsson C, Lorenzon M, Orwoll E, et al.Estimation of physical performance and measurements of habitual physical activity may capture men with high risk to fall--data from the Mr Os Sweden cohort. Arch Gerontol Geriatr 2009;49(1):e72-6.
11. Peeters GM, van Schoor NM, Pluijm SM, Deeg DJ, Lips P.Is there a U-shaped association between physical activity and falling in older persons? Osteoporos Int 2009;21(7):1189-95.

12. Delbaere K, Close JC, Heim J, Sachdev PS, Brodaty $\mathrm{H}$, Slavin MJ, et al.A multifactorial approach to understanding fall risk in older people.J Am Geriatr Soc 2010;58(9):1679-85.

13. Tinetti ME, Speechley M, Ginter SF.Risk factors for falls among elderly persons living in the community.N Engl J Med 1988;319(26):1701-7.

14. Ganz DA, Bao Y, Shekelle PG, Rubenstein LZ.Will my patient fall?Jama 2007;297(1):77-86.

15. Moreland J, Richardson J, Chan DH, O'Neill J, Bellissimo A, Grum RM, et al.Evidence-based guidelines for the secondary prevention of falls in older adults. Gerontology 2003;49(2):93-116.

16. Speechley M.Unintentional Falls in Older Adults: A Methodological Historical Review.Can J Aging 2011;1-12.

17. Brucki S, Nitrini R, P C, Bertolucci P, Okamoto I.Sugestões para o uso do mini-exame do estado mental no Brasil. Arquivos de Neuro-Psiquiatria 2003;61 (3-B):777-81.

18. Folstein MF, Folstein SE, McHugh PR."Mini-mental state". A practical method for grading the cognitive state of patients for the clinician.J Psychiatr Res 1975;12(3):189-98.

19. Lamb SE, Jorstad-Stein EC, Hauer K, Becker C.Development of a common outcome data set for fall injury prevention trials: the Prevention of Falls Network Europe consensus. J Am Geriatr Soc 2005;53(9):1618-22.

20. Podsiadlo D, Richardson S.The timed "Up \& Go": a test of basic functional mobility for frail elderly persons.J Am Geriatr Soc 1991;39(2):142-8.

21. Yesavage JA, Brink TL, Rose TL, Lum O, Huang $\mathrm{V}$, Adey M, et al.Development and validation of a geriatric depression screening scale: a preliminary report.J Psychiatr Res 1982;17(1):37-49. 
22. Paradela EM, Lourenco RA, Veras RP.Validation of geriatric depression scale in a general outpatient clinic.Rev Saude Publica 2005;39(6):918-23.

23. Ramos LR, Toniolo J, Cendoroglo MS, Garcia JT, Najas MS, Perracini M, et al.Two-year followup study of elderly residents in S. Paulo, Brazil: methodology and preliminary results. Rev Saude Publica 1998;32(5):397-407.

24. Lipschitz DA.Screening for nutritional status in the elderly.Prim Care 1994;21(1):55-67.

25. Lebrão ML, Laurenti R.Saúde, bem-estar e envelhecimento: o estudo SABE no Município de São Paulo.Rev Bras Epidemiol 2005;8(2):127-41.

26. Heesch KC, Byles JE, Brown WJ.Prospective association between physical activity and falls in community-dwelling older women.J Epidemiol Community Health 2008;62(5):421-6.

27. Gomes G CF, Diogo M, Neri A, Guariento M, Sousa M.Physical performance and number of falls in older adult fallers. Rev Bras Fisioter 2009;13(5):430-7.

28. Gama ZA, Gomez-Conesa A.Risk factors for falls in the elderly: systematic review.Rev Saude Publica 2008;42(5):946-56.

29. Rose DJ.Preventing falls among older adults: no "one size suits all" intervention strategy.J Rehabil Res Dev 2008;45(8):1153-66.

30. Carvalho Ade M, Coutinho Eda S.Dementia as risk factor for severe bone fractures among the elderly. Rev Saude Publica 2002;36(4):448-54.

31. Yogev-Seligmann G, Hausdorff JM, Giladi N.The role of executive function and attention in gait.Mov Disord 2008;23(3):329-42; quiz 472.
32. Coppin AK, Shumway-Cook A, Saczynski JS, Patel $\mathrm{KV}$, Ble A, Ferrucci L, et al.Association of executive function and performance of dual-task physical tests among older adults: analyses from the InChianti study. Age Ageing 2006;35(6):619-24.

33. Ble A, Volpato S, Zuliani G, Guralnik JM, Bandinelli S, Lauretani F, et al.Executive function correlates with walking speed in older persons: the InCHIANTI study.J Am Geriatr Soc 2005;53(3):410-5.

34. Verghese J, Holtzer R, Lipton RB, Wang C.Quantitative gait markers and incident fall risk in older adults.J Gerontol A Biol Sci Med Sci 2009;64(8):896-901.

35. Beauchet O, Annweiler C, Dubost V, Allali G, Kressig RW, Bridenbaugh S, et al.Stops walking when talking: a predictor of falls in older adults?Eur J Neurol 2009;16(7):786-95.

36. Rosano C, Brach J, Studenski S, Longstreth WT, Jr., Newman AB.Gait variability is associated with subclinical brain vascular abnormalities in highfunctioning older adults. Neuroepidemiology 2007;29(34):193-200.

37. Borges PLC, Bretas RP, Azevedo SF, Barbo JMM. Perfi 1 dos idosos freqüentadores de grupos de convivência em Belo Horizonte, Minas Gerais, Brasil.Cad Saúde Pública 2008;24(12):2798-808.

38. Silva HO, Carvalho MJAD, Lima FEL, Rodrigues LV.Perfil epidemiológico de idosos frequentadores de grupos de convivência no município de Iguatu, Ceará.Rev Bras Geriatr Gerontol 2011;14(1):123-33. 